\title{
Fokker-Planck linearization for non-Gaussian stochastic elastoplastic finite elements
}

\author{
Konstantinos Karapiperis ${ }^{\mathrm{a}, *}$, Kallol Sett ${ }^{\mathrm{b}}$, M. Levent $\operatorname{Kavvas}^{\mathrm{a}}$, Boris \\ Jeremić ${ }^{a, c}$ \\ ${ }^{a}$ Department of Civil and Environmental Engineering, University of California, Davis, \\ $C A, U S A$ \\ ${ }^{b}$ Department of Civil, Structural and Environmental Engineering, University at Buffalo, \\ The State University at New York, Buffalo, NY, USA \\ ${ }^{c}$ Earth Science Division, Lawrence Berkeley National Laboratory, Berkeley, CA, USA
}

\begin{abstract}
Presented here is a finite element framework for the solution of stochastic elastoplastic boundary value problems with non-Gaussian parametric uncertainty. The framework relies upon a stochastic Galerkin formulation, where the stiffness random field is decomposed using a multidimensional polynomial chaos expansion. At the constitutive level, a Fokker-Planck-Kolmogorov (FPK) plasticity framework is utilized, under the assumption of small strain kinematics. A linearization procedure is developed that serves to update the polynomial chaos coefficients of the expanded random stiffness in the elastoplastic regime, leading to a nonlinear least-squares optimization problem. The proposed framework is illustrated in a static shear beam example of elastic-perfectly plastic as well as isotropic hardening material.
\end{abstract}

Keywords: Fokker-Planck equation, elastoplasticity, stochastic finite elements, linearization, polynomial chaos, non-Gaussian

\footnotetext{
* Corresponding author

Email address: kkarapiperis@ucdavis.edu (Konstantinos Karapiperis)
}

Preprint submitted to Computer Methods in Applied Mechanics and Engineering February 29, 2016

(C) 2016. This manuscript version is made available under the Elsevier user license http://www.elsevier.com/open-access/userlicense/1.0/ 


\section{Introduction}

In constitutive modeling, material parameters are traditionally defined in a deterministic fashion by usually extracting the mean from a number of experiments. However, the behavior of all engineering materials, let alone

5 geomaterials, is inherently uncertain, as portrayed by various researchers [13]. The uncertain response follows from inherent uncertainty of material behavior and/or spatial non-uniformity of material distribution. In addition, the nonlinear material behavior present in several engineering applications is usually described using elastoplastic constitutive relations. The physical or phenomenological components of such a model are ideally described by random fields, most of which are non-Gaussian. Modeling them as Gaussian fields can induce both inaccuracy and instability to the solution of a boundary value problem. For example, a Gaussian representation of the material stiffness results in inaccurate higher order moments while physically allowing negative realizations of the process to occur (softening). To realistically approximate such a physical quantity, a strictly positive definite field is required.

Intrusive uncertainty quantification (UQ) frameworks, in which the uncertainty is propagated through the governing differential equations, are in many cases more efficient than non-intrusive ones. However, most researchers have focused on non-intrusive methods, which are easier to develop and utilize existing computational tools, or have limited their attention to intrusive UQ for simpler problems. The simplest example of a non-intrusive method is Monte Carlo Simulation (MCS) [4, 5], which may be seen as a direct integration 
method in which the integration points are chosen randomly over the probability space. Depending on the application, the latter approach can prove so computationally demanding that any practical application is hindered, at least for elasto-plastic models. Lately, more sophisticated sampling-based approaches have been developed including stochastic collocation [6, 7] and non-intrusive Galerkin techniques [8, 9]. The applicability of those methods is not affected by the complexity of the problem since they act as wrappers on a deterministic solver which in turn acts as a "black box".

Several researchers have dealt so far with intrusive uncertainty quantification in computational mechanics with an emphasis on linear problems. A comprehensive review of such methods may be found in [5, 10, 11], where the authors also provide insight to the well-posedness and structure of a stochastic boundary value problem. So far, the most widely used method for the quantification of uncertainty has been the stochastic finite element method (SFEM) [12, which relies on a spectral decomposition of parametric uncertainties and a polynomial chaos [13] approximation of the output random field. It is one of the first developments of a stochastic Galerkin method, where the problem is formulated in a variational form and holds in a weak sense. This class of methods allows for an explicit functional representation of the solution in terms of independent random variables. An overview of stochastic Galerkin methods may be found in [11, 14, 15]. A significant contribution to the efficiency of the above methods with respect to different classes of non-Gaussian processes has been the introduction of the generalized polynomial chaos expansion (gPCE) [16], guaranteeing optimal (exponential) convergence rates through an appropriate choice of orthogo- 
nal polynomials from the Askey family. Researchers also have attempted to address the curse of dimensionality associated with these methods by developing sparse approximations through low-rank tensor product techniques [17], proper generalized decompositions and separated representations [18].

The first attempt to extend SFEM to nonlinear material behavior was 55 by Anders and Hori [19], who used a perturbation expansion at the stochastic mean behavior. In computing the mean behavior they took advantage of bounding media approximation by introducing two fictitious bounding bodies providing an upper and a lower bound for the mean. This method, however, inherits the "closure problem" (essentially the need for higher order statistical moments in order to calculate lower order statistical moments) and suffers from the "small coefficient of variation" requirement for the material parameters. Later, Jeremić et al. 20] derived a second-order exact expression for the evolution of the probability density function of stress for elastoplastic constitutive rate equations with uncertain material parameters. Utilizing an Eulerian-Lagrangian form of the Fokker-Planck-Kolmogorov (FPK) equation [21], the aforementioned "closure problem" associated with regular perturbation methods is resolved. Afterwards, Jeremić and Sett [22] modified their approach to account for probabilistic rather than expected yielding and incorporated their developed FPK-based elastoplastic model in a Gaussian spectral stochastic finite element framework [23]. Later, Rosić [24] and Arnst and Ghanem [25] presented in detail the variational theory behind the mixed-hardening stochastic plasticity problem along with stochastic versions of relevant established computational plasticity algorithms.

In this paper, we utilize an FPK plasticity framework at the constitutive 
level and a stochastic Galerkin framework at the finite element level. NonGaussian parametric uncertainty is considered through a combined KarhunenLoève/polynomial chaos (KL/PC) expansion. The above are coupled through an FPK linearization scheme that updates the coefficients of the polynomial chaos (PC) approximation of the random stiffness. As opposed to the stochastic extension of classic variational inequality algorithms [24, 25], the FPK equation provides a way to transform the problem to a deterministic advection-diffusion equation and takes advantage of efficient algorithms developed for relevant problems. In addition, it helps overcome potential challenges associated with the accurate approximation of random inequality constrains, present in variational inequality methods (representation of convex elastic cones). Further, this method may be tailored to provide varying order of accuracy counterbalanced by computational efficiency through appropriate selection of the KL/PC spaces in which the constitutive integration procedure is performed. First, the stochastic approximation schemes are discussed followed by the finite element formulation. Next, the underlying FPK framework is introduced along with the proposed linearization procedure and the complete framework is illustrated with a simple static shear beam example.

\section{Stochastic discretization}

\subsection{Elastic stiffness}

Any arbitrary non-stationary stiffness random field may be approximated using a combined Karhunen-Loève/polynomial chaos methodology. This technique involves representation of an arbitrary stochastic process as a poly- 
nomial of a suitable underlying Gaussian field, whose covariance structure is decomposed by means of the Karhunen-Loève expansion (KLE). Following Sakamoto and Ghanem [26], we represent the uncertain elastic constitutive tensor field with the help of the polynomial chaos expansion (PCE):

$$
D(\mathbf{x}, \theta)=\sum_{i=0}^{M} r_{i}(\mathbf{x}) \Phi_{i}\left[\left\{\xi_{r}(\theta)\right\}\right]
$$

where $\Phi_{i}\left[\left\{\xi_{r}(\theta)\right\}\right]$ is a set of Hermite polynomials of an underlying Gaussian set $\xi_{r}(\theta)$ and $\theta$ is an element of the space of random variables $\Omega$. The variable $M$ denotes the order of the PCE. It can be shown that the latter is convergent in $L_{2}(\Omega)$; a relevant convergence rate study can be found in [27].

The spatially dependent coefficients $r_{i}$ may be computed via simple projection but this kind of expansion is defined without any reference to the random field $D(\mathbf{x}, \theta)$ and the expected accuracy is low. Therefore, a correlation structure is endowed to the underlying field by considering the following multidimensional PC representation:

$$
\left.D(\mathbf{x}, \theta)=\sum_{i=0}^{M} D_{i}(\mathbf{x}) \Gamma_{i}[\mathbf{x}, \theta)\right]
$$

where $\Gamma_{i}[\mathbf{x}, \theta]$ is now a set of multidimensional Hermite polynomials of an underlying correlated Gaussian field $\gamma(\mathbf{x}, \theta)$. The orthogonality of the polynomials is employed to calculate the coefficients $D_{i}(\mathbf{x})$ as:

$$
D_{i}(\mathbf{x})=\frac{\left\langle D \Gamma_{i}\right\rangle}{\left\langle\Gamma_{i}^{2}\right\rangle}
$$

where the numerator can be evaluated with the inverse CDF approach using some type of numerical quadrature (e.g., collocation, Monte Carlo (MC), 
quasi Monte Carlo (QMC), etc.). The correlation function $\rho_{D}$ of $\gamma(\mathbf{x}, \theta)$ induced on $D(\mathbf{x}, \theta)$ is given as the solution to the following polynomial equation [26]:

$$
\rho_{D}\left(\mathbf{x}_{1}, \mathbf{x}_{2}\right)=\sum_{i=1}^{M} D_{i}\left(\mathbf{x}_{1}\right) D_{i}\left(\mathbf{x}_{2}\right) i !\left\langle\gamma\left(\mathbf{x}_{1}\right) \gamma\left(\mathbf{x}_{2}\right)\right\rangle^{i}
$$

120

$\mathrm{w}$

where $\mathbf{x}_{1}, \mathbf{x}_{2}$ denote two spatial points in the domain of interest. This equation is solved by discretizing the domain into a number of nodes and solving the resulting system of equations with the following constraint:

$$
\frac{\partial \rho_{D}\left(\mathbf{x}_{1}, \mathbf{x}_{2}\right)}{\partial\left\langle\gamma\left(\mathbf{x}_{1}\right) \gamma\left(\mathbf{x}_{2}\right)\right\rangle}=\sum_{i=1}^{M} i \frac{i ! D_{i}^{2}}{\sum_{j=1}^{M} j ! D_{j}^{2}}\left\langle\gamma\left(\mathbf{x}_{1}\right) \gamma\left(\mathbf{x}_{2}\right)\right\rangle^{i-1}>0
$$

Knowing the above, the correlated random field $\gamma(\mathbf{x}, \theta)$ may be expanded in the following Karhunen-Loève form:

$$
\gamma(\mathbf{x}, \theta)=\sum_{i=1}^{Q} \sqrt{\lambda_{i}} f_{i}(\mathbf{x}) \xi_{i}(\theta)
$$

subject to the following constraint deriving from the unit variance condition imposed on $\gamma(\mathbf{x})$ :

$$
\sum_{i=1}^{Q}\left(\sqrt{\lambda_{i}} f_{i}(\mathbf{x})\right)^{2}=1
$$

In the above equations, $\lambda_{i}$ and $f_{i}(\mathbf{x})$ denote the resulting eigenvalues and eigenvectors respectively, and $Q$ denotes the dimensionality of the truncated expansion. It is required that we re-normalize to a unit variance as follows:

$$
\gamma(\mathbf{x}, \theta)=\sum_{i=1}^{Q} \frac{\sqrt{\lambda_{i}} f_{i}(x)}{\sqrt{\sum_{m=1}^{Q}\left(\sqrt{\lambda_{m}} f_{m}(\mathbf{x})\right)^{2}}} \xi_{i}(\theta)
$$


130

can find the coefficients $r_{i}(x)$ as

$$
r_{i}(\mathbf{x})=\frac{p !}{\left\langle\Phi_{i}^{2}\right\rangle} D_{p}(\mathbf{x}) \prod_{j=1}^{p} \frac{\sqrt{\lambda_{k(j)}} f_{k(j)}(\mathbf{x})}{\sqrt{\sum_{m=1}^{Q}\left(\sqrt{\lambda_{m}} f_{m}(\mathbf{x})\right)^{2}}}
$$

where $p$ is the order of the polynomial $\Phi_{i}$ and $k$ is an index on at least one of the $\xi_{k}$ making up $\Phi_{i}$. Note that the accuracy of the synthesized marginal probability density function depends mainly on the order $M$ of the PC expansion, while the correlation accuracy depends on the dimension $Q$ of $\xi(\theta)$. In the case of arbitrary non-Gaussian processes, the suitability of KLE as a means of characterizing the random variables entering the PCE or gPCE [16] is questionable. This is because, in this case, KLE yields uncorrelated but dependent random variables. Adaptation of the general PC framework to suitably chosen probability measures is presented in [28]. Often, in this case, transformation techniques such as the Rosenblatt [29] or the Nataf [30] tranform are utilized.

This study assumes a strictly positive definite lognormal random stiffness field in conjunction with classical PCE [31], which admit the analytical com145 putation of the respective coefficient (rather than the numerical techniques described above). Assuming an underlying Gaussian field $g(\mathbf{x})$, the actual stiffness field is given by:

$$
D(\mathbf{x})=e^{g(\mathbf{x})}
$$

with the following mean and variance relations:

$$
\begin{aligned}
\bar{D} & =e^{\bar{g}} \\
\sigma_{D} & =e^{\sigma_{g}}
\end{aligned}
$$


The process $g(\mathbf{x})$ is expanded in the Karhunen-Loève sense as:

$$
g(\mathbf{x})=\bar{g}+\sum_{i=1}^{N} g_{i} \xi_{i}=\bar{g}+\sum_{i=1}^{N} \sqrt{\lambda_{i}} f_{i}(\mathbf{x}) \xi_{i}
$$

150

and projection into polynomial chaos yields analytical coefficients $r_{i}(\mathbf{x})$ :

$$
r_{i}(\mathbf{x})=\frac{\left\langle e^{g} \Phi_{i}\right\rangle}{\left\langle\Phi_{i}^{2}\right\rangle}=\frac{\prod_{j=1}^{p} \sqrt{\lambda_{k(j)}} f_{k(j)}(\mathbf{x})}{\left\langle\Phi_{i}^{2}\right\rangle} e^{\bar{g}+\frac{1}{2} \sum_{j=1}^{N} g_{j}^{2}}
$$

Fig. 1] shows how the synthesized marginal probability density function using this methodology converges to the target lognormal distribution for a case of $\mathrm{COV}=30 \%$ for an increasing order of polynomial chaos approximation. Fig. 2, on the other hand, compares the target and approximated correlation structure for varying KL dimensionality.

\subsection{Shear strength}

In the case of a non-Gaussian shear strength random field $S_{u}(\mathbf{x}, \theta)$, the above methodology may be applied considering the two fields to be independent of each other. Alternatively, for computational efficiency, one may go with with a Gaussian random field. In this case, however, care should be taken so that the shear strength remains bounded and positive in order to ensure physical behaviour and well-posedness of the problem [32]. Note that this choice may impose a tight restriction on the applicable coefficient of variation. Then, the shear strength field may be approximated simply by a KLE as follows:

$$
S_{u}(\mathbf{x}, \theta)=\bar{S}_{u}(\mathbf{x})+\sum_{i=1}^{N} \sqrt{\lambda_{i}} f_{i}(\mathbf{x}) \xi_{i}(\theta)
$$



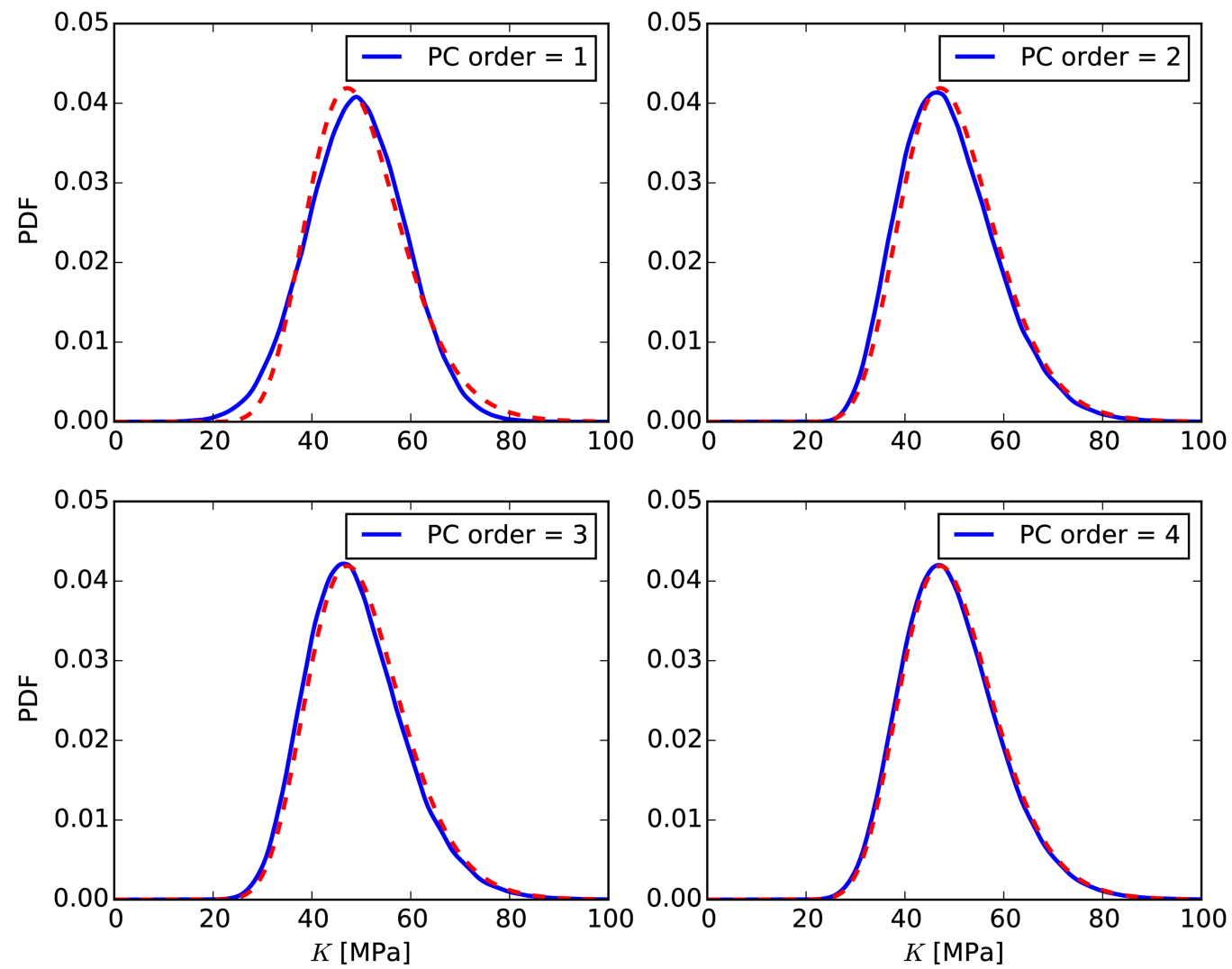

Figure 1: Convergence of the PC approximation (blue) to the target (red) lognormal distribution. 


\section{$\mathrm{KL} \operatorname{dim}=2$}

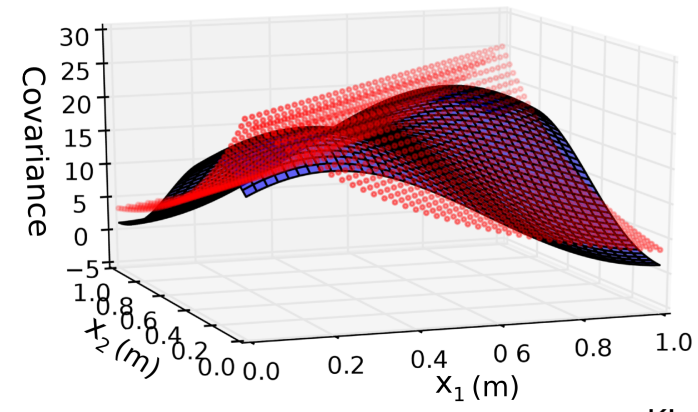

\section{$\mathrm{KL} \operatorname{dim}=10$}

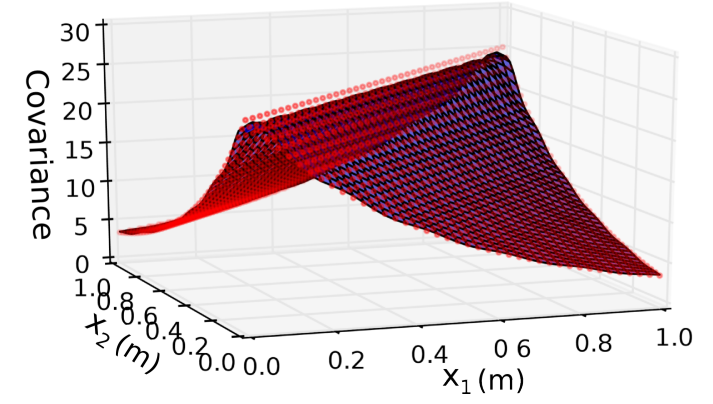

Figure 2: Comparisons of the approximated (blue) and the target (red) correlation structures for varying KL dimensionality.
$\mathrm{KL} \operatorname{dim}=5$

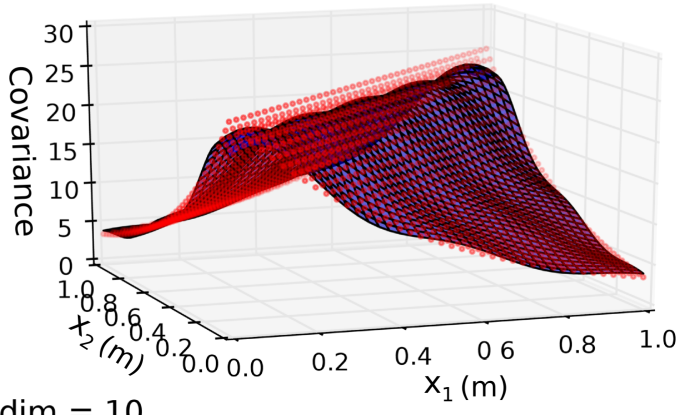


by considering the following Fredholm integral equation of the second kind [33] with the covariance function $C_{S_{u}}$ as a kernel:

$$
\int_{D} C_{S_{u}}\left(\mathbf{x}_{1}, \mathbf{x}_{2}\right) f_{k}\left(\mathbf{x}_{1}\right) d \mathbf{x}_{1}=\lambda_{k} f_{k}\left(\mathbf{x}_{2}\right)
$$

This expansion is optimal in the sense that it is the best approximation that may be achieved in the $L_{2}(D) \otimes L_{2}(\Omega)$ norm.

170 In some cases (e.g., triangular, exponential kernel) the above eigenproblem may be solved analytically, but in the general case a numerical approximation scheme is required. In that sense, a number of methods have been applied including FEM [12], wavelet-Galerkin [34, $\mathcal{H}$-matrices [35] and meshless methods [36].

In a standard finite element setting, each eigenfunction, $f_{k}$ of the kernel is approximated as:

$$
f_{k}(\mathbf{x})=\sum_{i=1}^{N} d_{i k} h_{i}(\mathbf{x})
$$

where $h$ and $d$ represent basis functions of compact support and appropriate nodal coefficients, respectively. Utilizing the above representation and requiring the error to be orthogonal to the approximating space, one may transform Eq. (16) to the following weak form:

$$
\sum_{i=1}^{N} d_{i k}\left[\int_{D} \int_{D} C_{S_{u}}\left(\mathbf{x}_{1}, \mathbf{x}_{2}\right) h_{i}\left(\mathbf{x}_{2}\right) h_{j}\left(\mathbf{x}_{1}\right) d \mathbf{x}_{1} d \mathbf{x}_{2}-\lambda_{k} \int_{D} h_{i}(\mathbf{x}) h_{j}(\mathbf{x}) d \mathbf{x}\right]=0
$$

The required discretization (mesh size) depends on the correlation length describing the rate of fluctuation of the random field. It has been shown [37, 38] that 2-4 elements per correlation length are usually enough to capture the structure of the random field. For example, a 1-dimensional $10 \mathrm{~m}$ 
long domain requires a 20-40 element mesh for $l_{c}=1 \mathrm{~m}$, while only 2 -4 elements are adequate for $l_{c}=10 \mathrm{~m}$. In cases where the correlation structure is approximated by long-tailed kernels (e.g., Gaussian), the resulting generalized "stiffness" matrix in the eigenproblem looses its sparsity resulting in an inefficient numerical solution. It is therefore common to modify (truncate) the kernels so as to increase the sparsity of the representation. Melink and Korelc [39] studied this problem in terms of numerical integration and loss of positive definiteness of the covariance matrix.

\section{Spatial and Stochastic discretization of the solution}

The unknown displacement random field is semi-discretized in the stochastic dimension using PCE:

$$
u(\mathbf{x}, \theta)=\sum_{i=0}^{P} d_{i}(\mathbf{x}) \Psi_{i}\left[\xi_{r}(\theta)\right]
$$

where $\Psi_{i}\left[\xi_{r}(\theta)\right]$ represents a set of random Hermite polynomials of order $P$. The component $d_{i}(\mathbf{x})$ is, then, further discretized in the spatial sense using standard finite element shape functions:

$$
d_{i}(\mathbf{x})=\sum_{j=1}^{N} d_{i j} N_{j}(\mathbf{x})
$$

This results in a final expression for the random displacement field:

$$
u(\mathbf{x}, \theta)=\sum_{i=0}^{P} \sum_{j=1}^{N} d_{i j} N_{j}(\mathbf{x}) \Psi_{i}\left[\xi_{r}(\theta)\right]
$$


200

\section{Finite Element Formulation}

Employing the Galerkin weak formulation of linearized static FEM [40], we have the following simplified form :

$$
\sum_{e}\left[\int_{D_{e}} \nabla N_{m}(\mathbf{x}) D(\mathbf{x}, \theta) \nabla N_{n}(\mathbf{x}) d V u_{n}-\int_{D_{e}} f_{m}(\mathbf{x}, \theta) d V\right]=0
$$

where $\sum_{e}$ denotes the assembly procedure over all finite elements of the discretized domain $V$ and $f_{m}(\mathbf{x})$ incorporates the various elemental contribu205 tions to the global force vector.

Combining Equations (1), 21) and (22) and denoting the shape function gradients as:

$$
\nabla N_{n}(\mathbf{x}):=B_{n}(\mathbf{x})
$$

yields:

$$
\begin{aligned}
\sum_{e} & {\left[\int_{D_{e}} B_{m}(\mathbf{x}) \sum_{i=0}^{M} r_{i}(\mathbf{x}) \Phi_{i}\left[\left\{\xi_{r}(\theta)\right\}\right] B_{n}(\mathbf{x}) \sum_{j=0}^{P} d_{n j} \Psi_{j}\left[\xi_{r}(\theta)\right] d V\right.} \\
& \left.-\int_{D_{e}} f_{m}(\mathbf{x}, \theta) d V\right]=0
\end{aligned}
$$

Taking now the Galerkin projection of the discretized equation onto each 210 arbitrary polynomial basis of the displacement approximation $\Psi_{k}\left[\xi_{r}(\theta)\right]$ :

$$
\begin{aligned}
\sum_{e} & {\left[\int_{D_{e}} B_{m}(\mathbf{x}) \sum_{i=0}^{M} r_{i}(\mathbf{x}) \Phi_{i}\left[\left\{\xi_{r}(\theta)\right\}\right] B_{n}(\mathbf{x}) \sum_{j=0}^{P} \sum_{k=0}^{P} d_{n j} \Psi_{j}\left[\xi_{r}(\theta)\right] \Psi_{k}\left[\xi_{r}(\theta)\right] d V\right.} \\
& \left.-\int_{D_{e}} \sum_{k=0}^{P} f_{m}(\mathbf{x}, \theta) \Psi_{k}\left[\xi_{r}(\theta)\right] d V\right]=0
\end{aligned}
$$

Taking expectation on both sides results in the following system of equations:

$$
\sum_{n=1}^{N} \sum_{j=0}^{P} d_{n j} \sum_{k=1}^{M} b_{i j k} K_{m n i}=F_{m}\left\langle\Psi_{k}\left[\left\{\xi_{r}\right\}\right]\right\rangle
$$


where

$$
K_{m n i}=\int_{D} B_{m}(\mathbf{x}) r_{i}(\mathbf{x}) B_{n}(\mathbf{x}) d V
$$

and

$$
F_{m}=\int_{D} f_{m}(\mathbf{x}, \theta) d V
$$

Symbolic manipulations are carried out using Mathematica 41] in order to precompute the coefficients of the tensor:

$$
b_{i j k}=\left\langle\Phi_{i}\left[\left\{\xi_{r}\right\}\right] \Psi_{j}\left[\left\{\xi_{r}\right\}\right] \Psi_{k}\left[\left\{\xi_{r}\right\}\right]\right\rangle
$$

The form of the latter induces a special block sparsity in the resulting stiffness matrix that may be exploited to develop an efficient solution scheme. Several researchers have dealt with such systems of equations arising in the context of the the spectral stochastic finite element formulation. One of the first attempts was made by Ghanem and Kruger [42] who proposed two solution procedures, a preconditioned CG method as well as a hierarchical formulation. Another iterative scheme of the family of Krylov-subspace methods that has been applied is the preconditioned MINRES [43]. In addition, researchers have developed multi-grid approaches [44] as well as incomplete block-diagonal preconditioning schemes based on the FETI-PD solver [45]. A more complete review of the methods may be found in [24].

\section{Elastoplasticity}

In this study, the elastoplastic behavior is treated in a spectral fashion by updating the coefficients of the stochastic approximation of the stiffness 


$$
\frac{d \sigma_{i j}\left(x_{t}, t\right)}{d t}=\beta_{i j k l}\left(\sigma_{i j}, D_{i j k l}, q_{*}, r_{*} ; x_{t}, t\right) \frac{d \epsilon_{k l}\left(x_{t}, t\right)}{d t}
$$


or

$$
\frac{d \sigma_{i j}\left(x_{t}, t\right)}{d t}=\eta_{i j k l}\left(\sigma_{i j}, D_{i j k l}, \epsilon_{k l}\left(x_{t}, t\right) q_{*}, r_{*} ; x_{t}, t\right)
$$

where the stochasticity of the operator $\beta$ is induced by the stochasticity of $D_{i j k l}, q_{*}, r_{*}$. This renders the above equation a linear/non-linear ordinary dif-

250

where $N_{(1)}$ and $N_{(2)}$ are advection and diffusion coefficients respectively that are particular to the constitutive model. Given the initial and boundary 
conditions as well as the second-order statistics of material properties, Eq. (35) may be solved with second-order accuracy.

To account for the uncertainty in the probabilistic yielding, Jeremić and Sett [22] introduced the following equivalent advection and diffusion coefficients:

$$
\begin{aligned}
N_{(1)_{m n}}^{\sigma^{e q}}\left(\sigma_{i j}\right) & =(1-P[f>0]) N_{(1)_{m n}}^{e l}+P[f>0] N_{(1)_{m n}}^{e p} \\
N_{(2)_{m n a b}}^{\sigma^{e q}}\left(\sigma_{i j}\right) & =(1-P[f>0]) N_{(2)_{m n a b}}^{e l}+P[f>0] N_{(2)_{m n a b}}^{e p}
\end{aligned}
$$

where $(1-P[f>0])$ represents the probability of the material being elastic, while $P[f>0]$ represents the probability of the material being elastoplastic. $P[f>0]$ is obtained from the cumulative density function, rendering it an explicit function of the stress $\sigma_{i j}$ as well as the internal variables $q_{*}$.

Utilizing Eq. (34), one may compute the elastic and elastoplastic coefficients addressed in Eq. (35) as:

$$
\begin{aligned}
N_{(1)_{m n}}^{e l} & =\left\langle D_{m n r s}^{e l} \dot{\epsilon}_{r s}\right\rangle \\
N_{(2)_{m n a b}}^{e l} & =t \operatorname{Cov}_{0}\left[D_{m n r s}^{e l} \dot{\epsilon}_{r s} ; D_{a b c d}^{e l} \dot{\epsilon}_{c d}\right]
\end{aligned}
$$

and

$$
\begin{aligned}
N_{(1)_{m n}}^{e p} & =\left\langle D_{m n r s}^{e p} \dot{\epsilon}_{r s}\right\rangle+\int_{0}^{t} d \tau \operatorname{Cov}_{0}\left[\frac{\partial}{\partial \sigma_{a b}}\left\{D_{m n r s}^{e p} \dot{\epsilon}_{r s}\right\} ; D_{a b c d}^{e p} \dot{\epsilon}_{c d}\right] \\
N_{(2)_{m n a b}}^{e p} & =\int_{0}^{t} d \tau \operatorname{Cov}_{0}\left[D_{m n r s}^{e p}(t) \dot{\epsilon}_{r s} ; D_{a b c d}^{e p}(t-\tau) \dot{\epsilon}_{c d}\right]
\end{aligned}
$$

The evolution of any internal variable $\mathbf{q}_{i}$ of the model is handled through 275 a coupled FPK equation of the form:

$$
\frac{\partial \tilde{P}\left(q_{i}, t\right)}{\partial t}=-\frac{\partial}{\partial q_{m}}\left[N_{(1)_{m}}^{q^{e q}}\left(\sigma_{m n}, q_{m}\right) \tilde{P}\left(q_{i}, t\right)-\frac{\partial}{\partial q_{n}}\left\{N_{(2)_{m n}}^{q^{e q}}\left(\sigma_{m n}, q_{m}\right) \tilde{P}\left(q_{i}, t\right)\right\}\right]
$$


The advection and diffusion coefficients in the above equation are given similarly to Equations (36) and (36) but with no contributions of any "elastic" state:

$$
\begin{aligned}
N_{(1)_{m}}^{q^{e q}}\left(\sigma_{i j}, q_{i}\right) & =P[f>0] N_{(1)_{m}}^{q^{e p}} \\
N_{(2)_{m n}}^{q^{e q}}\left(\sigma_{i j}, q_{i}\right) & =P[f>0] N_{(2)_{m n}}^{q^{e p}}
\end{aligned}
$$

The elastoplastic components of the equivalent advection and diffusion terms are functions of the so-called loading index or plastic multiplier $L$ and the rates of evolution of the internal variables $r_{i}$ :

$$
\begin{aligned}
N_{(1)_{m}}^{q^{e p}} & =\left\langle L r_{i}\right\rangle+\int_{0}^{t} d \tau \operatorname{Cov}_{0}\left[\frac{\partial}{\partial q_{j}} L r_{i}(t) ; L r_{j}(t-\tau)\right] \\
N_{(2)_{m n}}^{q^{e p}} & =\int_{0}^{t} d \tau \operatorname{Cov}_{0}\left[L r_{i}(t) ; L r_{j}(t-\tau)\right]
\end{aligned}
$$

where $L$ may be expressed as:

$$
L=\frac{\frac{\partial f}{\partial \sigma_{o t}} D_{i j o t}^{e l} \dot{\epsilon}_{i j}}{\frac{\partial U}{\partial \sigma_{a b}} D_{a b c d}^{e l} \frac{\partial f}{\partial \sigma_{c d}}-\frac{\partial f}{\partial q_{m}} r_{m}}
$$

\subsection{Linearization for stiffness update}

The constitutive integration of the FPK-based plasticity model cannot directly provide the updated generalized stiffness at the finite element level. Therefore, a numerical scheme is required in order to compute the stiffness in a PC expansion form as per Eq. (1). In this study we assume an equivalent linear FPK equation involving the updated PC coefficients which are deduced through a least-squares optimization procedure. 
For each orthogonal PC space, s, Eq. (35) applies with the advection and diffusion coefficients taking the form:

$$
\begin{aligned}
N_{(1)_{m n}}^{s^{e q}}\left(\sigma_{i j}^{s}, \mathbf{x}\right) & =(1-P[f>0]) N_{(1)_{m n}}^{e l}+P[f>0] N_{(1)_{m n}}^{e p} \\
N_{(2)_{\text {mnab }}}^{s^{e q}}\left(\sigma_{i j}^{s}, \mathbf{x}\right) & =(1-P[f>0]) N_{(2)_{\text {mnab }}}^{e l}+P[f>0] N_{(2)_{\text {mnab }}^{e p}}^{e p}
\end{aligned}
$$

For the purposes of this study, let us consider an isotropic linear elastic - Mises isotropic hardening model and derive the equivalent advection and diffusion coefficients for this case. The isotropic linear elasticity tensor in

$$
D_{i j k l}^{e l}=G \delta_{i k} \delta_{j l}+\left(K-\frac{2}{3} G\right) \delta_{i j} \delta_{k l}
$$

where $K$ and $G$ denote the bulk and shear modulus respectively and are represented as random fields (see for example Eq. (1)).

The elastoplastic continuum tangent tensor in Eq. (31) is given in the following general form:

$$
D_{i j k l}^{e p}=G \delta_{i k} \delta_{j l}+\left(K-\frac{2}{3} G\right) \delta_{i j} \delta_{k l}-\frac{A_{i j} A_{k l}^{*}}{B+K_{P}}
$$

300

The Mises linear hardening yield function is written as:

$$
f=\sqrt{J_{2}}-S_{u}=\sqrt{\frac{1}{2} s_{i j} s_{i j}}-S_{u}
$$

Under the assumption of associated flow rule we have:

$$
\frac{\partial f}{\partial \sigma_{i j}}=\frac{\partial U}{\partial \sigma_{i j}}
$$

which results in the following symmetry:

$$
A_{i j}=A_{i j}^{*}=D_{i j k l}^{e l} \frac{\partial f}{\partial \sigma_{k l}}
$$


After some algebraic manipulations, one can easily derive:

$$
A_{i j}=\frac{G}{\sqrt{J_{2}}} s_{i j} \quad B=G
$$

The plastic modulus $K_{P}$ is computed here on the basis of a deterministic

hardening rule in terms of the equivalent plastic strain:

$$
S_{u}=S_{u}\left(\epsilon_{e q}^{p}\right)
$$

After imposing the consistency condition, we have:

$$
K_{P}=-\frac{\partial f}{\partial S_{u}} \bar{k}=-\frac{1}{\sqrt{3}} \frac{\partial f}{\partial S_{u}} \frac{d S_{u}}{d \epsilon_{e q}^{p}} \frac{\partial f}{\partial \sqrt{J_{2}}}=\frac{1}{\sqrt{3}} \frac{d S_{u}}{d \epsilon_{e q}^{p}}
$$

Combining Equations (36)-(41) and (50)- (51), we can derive the final coefficients of the FPK constitutive rate equation as:

$$
\begin{aligned}
& N_{(1)_{m n}}^{s^{e q}}=(1-P[f>0])\left\langle\left[G \delta_{m r} \delta_{n s}+\left(K-\frac{2}{3} G\right) \delta_{m n} \delta_{r s}\right] \dot{\epsilon}_{r s}(t)\right\rangle \\
& +P[f>0]\left\langle\left[ G \delta_{m r} \delta_{n s}+\left(K-\frac{2}{3} G\right) \delta_{m n} \delta_{r s}-\right.\right. \\
& \left.\left.\frac{1}{G+\frac{1}{\sqrt{3}} \frac{d S_{u}}{d \epsilon_{e q}^{p}}}\left(\frac{G}{\sqrt{J_{2}}}\right)^{2} s_{i j}^{s}(t) s_{k l}^{s}(t)\right] \dot{\epsilon}_{r s}(t)\right\rangle \\
& +\int_{0}^{t} d \tau \operatorname{Cov}_{0}\left[\frac { \partial } { \partial \sigma _ { a b } ^ { s } } \left\{\left[G \delta_{m r} \delta_{n s}+\left(K-\frac{2}{3} G\right) \delta_{m n} \delta_{r s}\right.\right.\right. \\
& \left.\left.-\frac{1}{G+\frac{1}{\sqrt{3}} \frac{d S_{u}}{d \epsilon_{e q}^{p}}}\left(\frac{G}{\sqrt{J_{2}}}\right)^{2} s_{i j}^{s}(t) s_{k l}^{s}(t)\right] \dot{\epsilon}_{r s}(t)\right\} \text {; } \\
& {\left[G \delta_{a c} \delta_{b d}+\left(K-\frac{2}{3} G\right) \delta_{a b} \delta_{c d}-\right.} \\
& \left.\left.\frac{1}{G+\frac{1}{\sqrt{3}} \frac{d S_{u}}{d \epsilon_{e q}^{p}}}\left(\frac{G}{\sqrt{J_{2}}}\right)^{2} s_{a b}^{s}(t-\tau) s_{c d}^{s}(t-\tau)\right] \dot{\epsilon}_{c d}(t-\tau)\right]
\end{aligned}
$$




$$
\begin{aligned}
N_{(2)_{m n a b}^{s^{e q}}=} & (1-P[f>0]) t \operatorname{Cov}_{0}\left[\left\{G \delta_{m r} \delta_{n s}+\left(K-\frac{2}{3} G\right) \delta_{m n} \delta_{r s}\right.\right. \\
& \left.-\frac{1}{G+\frac{1}{\sqrt{3}} \frac{d S_{u}}{d \epsilon_{e q}^{p}}}\left(\frac{G}{\sqrt{J_{2}}}\right)^{2} s_{i j}^{s}(t) s_{k l}^{s}(t)\right\} \dot{\epsilon}_{r s}(t) ; \\
& {\left[G \delta_{a c} \delta_{b d}+\left(K-\frac{2}{3} G\right) \delta_{a b} \delta_{c d}-\right.} \\
& \left.\left.\frac{1}{G+\frac{1}{\sqrt{3}} \frac{d S_{u}}{d \epsilon_{e q}^{p}}}\left(\frac{G}{\sqrt{J_{2}}}\right)^{2} s_{a b}^{s}(t) s_{c d}^{s}(t)\right] \dot{\epsilon}_{c d}(t)\right] \\
& +P[f>0] \int_{0}^{t}\left(\frac { d \tau C v _ { 0 } } { } \left[\left\{\delta_{m r} \delta_{n s}+\left(K-\frac{2}{3} G\right) \delta_{m n} \delta_{r s}\right.\right.\right. \\
& \left.-\frac{1}{G+\frac{1}{\sqrt{3}} \frac{d S_{u}}{d \epsilon_{e q}^{p}}}\left(\frac{G}{\sqrt{J_{2}}}\right)^{2} s_{i j}^{s}(t) s_{k l}^{s}(t)\right\} \dot{\epsilon}_{r s}(t) ; \\
& \left\{G \delta_{a c} \delta_{b d}+\left(K-\frac{2}{3} G\right) \delta_{a b} \delta_{c d}-\right. \\
& \left.\left.\frac{1}{G+\frac{1}{\sqrt{3}} \frac{d S_{u}}{d \epsilon_{e q}^{p}}}\left(\frac{G}{\sqrt{J_{2}}}\right)^{2} s_{a b}^{s}(t-\tau) s_{c d}^{s}(t-\tau)\right\} \dot{\epsilon}_{c d}(t-\tau)\right]
\end{aligned}
$$

The solution of Eq. (35) may only provide the rate of change of the 310 probability density at the $\mathrm{k}^{\text {th }}$-step, yet not the updated stiffness at the finite element level. To deduce the latter, let us consider a linearized FPK equation for the stress corresponding to the orthogonal space $s$ at the same computational step in the following form:

$$
\frac{\partial P^{l i n}\left(\sigma_{i j}^{s}, t\right)}{\partial t}=-N_{(1)_{m n}}^{s^{l i n}} \frac{\partial P\left(\sigma_{i j}^{s}, t\right)}{\partial \sigma_{m n}^{s}}+N_{(2)_{m n a b}}^{s^{l i n}} \frac{\partial^{2} P\left(\sigma_{i j}^{s}, t\right)}{\partial \sigma_{m n}^{s} \sigma_{a b}^{s}}
$$


where the linearized advection and diffusion coefficients are given by:

$$
\begin{aligned}
& N_{(1)_{m n}}^{s^{l i n}}=r_{m n a b}^{s^{(k)}} \sum_{i=0}^{P}\left\langle\Phi_{i} \Psi_{s}\right\rangle \\
& \frac{1}{2 \Delta t} \sum_{j=1}^{N}\left[N_{j, b}(\mathbf{x}) \Delta d_{i j a}^{k-1}+N_{j, a}(\mathbf{x}) \Delta d_{i j b}^{k-1}\right] \\
& N_{(2)_{m n a b}}^{s^{l i n}}=t\left(r_{m n a b}^{s^{(k)}}\right)^{2} \sum_{i=0}^{P} \operatorname{Var}\left[\Phi_{i} \Psi_{s}\right] \\
& \frac{1}{4 \Delta t^{2}}\left[N_{j, b}(\mathbf{x}) \Delta d_{i j a}^{k-1}+N_{j, a}(\mathbf{x}) \Delta d_{i j b}^{k-1}\right]^{2}
\end{aligned}
$$

In an explicit scheme, the strain increment at the $(\mathrm{k}-1)^{\text {th }}$ step is utilized, while the fourth-order tensor valued PC coefficient $r_{m n a b}^{s^{(k)}}$ is unknown. Depending on the specific constitutive model, the above equations may be simplified to include scalar PC coefficients and deterministic bases in an appropriate tensor format. Combining Equations (35) and 60), one ends up with an over-determined residual system of equations in terms of the unknown coefficients at time step $k$ :

$$
R_{i}\left(r_{m n a b}^{s^{(k)}}\right)=\frac{\partial P^{l i n}\left(\boldsymbol{\sigma}_{i}^{s}, t\right)}{\partial t}-\frac{\partial P\left(\boldsymbol{\sigma}_{i}^{s}, t\right)}{\partial t}=0, \quad i=1, \ldots, N
$$

Each equation corresponds to a single point in the stress domain and the system of equations may be solved in the least squares sense using, for example, the Levenberg - Marquardt algorithm [46]. Therefore, a stiffness update procedure is established. A summary of the constitutive update algorithm is given in Algorithm 1 .

\subsection{Varying order of accuracy}

The outlined linearization scheme is accurate to the order of the PC approximation of the stiffness. However, one can restrict the accuracy of 


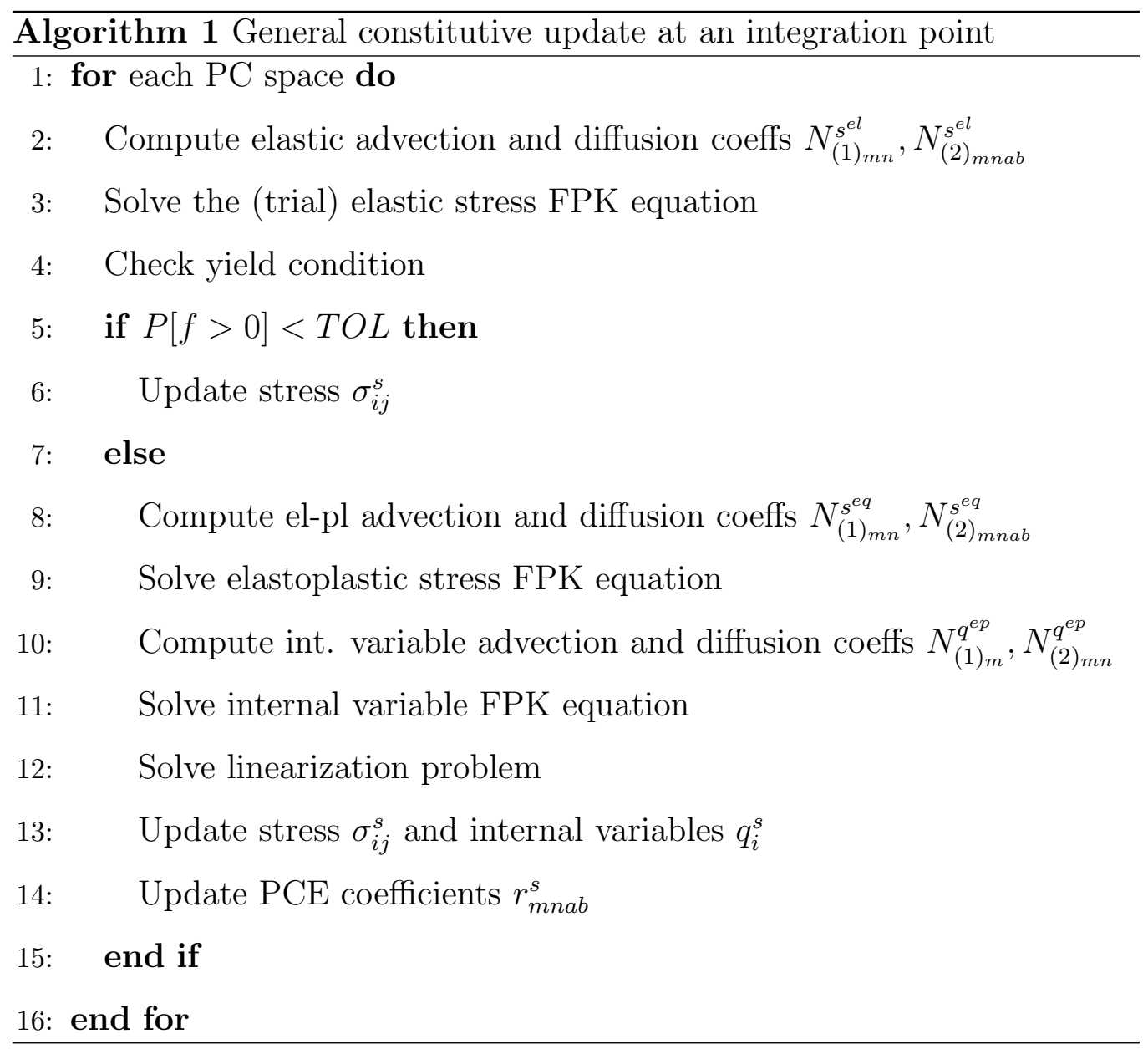

the method to second order with significant computational time savings, by considering the integration of a single FPK equation at any point in the discretized domain. This is achieved by considering the total stress, $\boldsymbol{\sigma}$ rather than each PC stress component, $\boldsymbol{\sigma}^{s}$. The linearized equation becomes:

$$
\frac{\partial P^{l i n}\left(\sigma_{i j}, t\right)}{\partial t}=-N_{(1)}^{l i n} \frac{\partial P\left(\sigma_{i j}, t\right)}{\partial \sigma_{m n}}+N_{(2)}^{l i n} \frac{\partial^{2} P\left(\sigma_{i j}, t\right)}{\partial \sigma_{m n} \sigma_{a b}}
$$


where:

$$
\begin{aligned}
N_{(1)}^{l i n}= & \sum_{s=0}^{M} r_{m n a b}^{s^{(k)}} \sum_{i=0}^{P}\left\langle\Phi_{i} \Psi_{s}\right\rangle \\
& \frac{1}{2 \Delta t} \sum_{j=1}^{N}\left[N_{j, b}(\mathbf{x}) \Delta d_{i j a}^{k-1}+N_{j, a}(\mathbf{x}) \Delta d_{i j b}^{k-1}\right] \\
N_{(2)}^{l i n}\left(r_{s}^{k}, \mathbf{x}\right)= & t \sum_{s=0}^{M}\left(r_{m n a b}^{s^{(k)}}\right)^{2} \sum_{i=0}^{P} \operatorname{Var}\left[\Phi_{i} \Psi_{s}\right] \\
& \frac{1}{4 \Delta t^{2}}\left[N_{j, b}(\mathbf{x}) \Delta d_{i j a}^{k-1}+N_{j, a}(\mathbf{x}) \Delta d_{i j b}^{k-1}\right]^{2}
\end{aligned}
$$

Again, the resulting system of equations is generally over-determined and may be solved for $r_{m n a b}^{s^{(k)}}$ using least-squares techniques. Due to the form of the FPK constitutive integrator, we do not expect higher order accuracy in the linearized tangent stiffness. Indeed, the Gaussian nature of this second-order exact variation of the linearization procedure, suggests that the advection and diffusion coefficients at each integration point may be fully described by two independent coefficients $\left(r_{m n a b}^{s^{(k)}}, s=1,2\right)$. This implies that the polynomial chaos coefficients that correspond to third and higher order $\left(r_{m n a b}^{s^{(k)}}, s \geq 3\right)$ will be dependent (negatively correlated) variables. It is proposed that the higher order coefficients retain their elastic (initial) values in order to achieve higher order accuracy during elastic loading or unloading.

In a more general sense, in order to achieve higher than second order accuracy and at the same time save on computation compared to the procedure proposed in Section 5.2 , the integration procedure may be applied to a restricted number of orthogonal PC spaces. The set of spaces in which the integration procedure is eventually carried out may be chosen on the basis of a posteriori error estimation techniques. However, a study of the accuracy 
of such a framework is out of the scope of this study.

\section{Numerical illustrations}

In this last section, the proposed framework is applied to the static loading of a shear beam representing a one-dimensional soil column under undrained conditions. The idealized numerical model is shown in Fig. 3. Two different cases are considered to test the methodology against parameters that differentiate the contribution of each orthogonal space, the evolution of the stress PDF as well as the global response (Table 1).

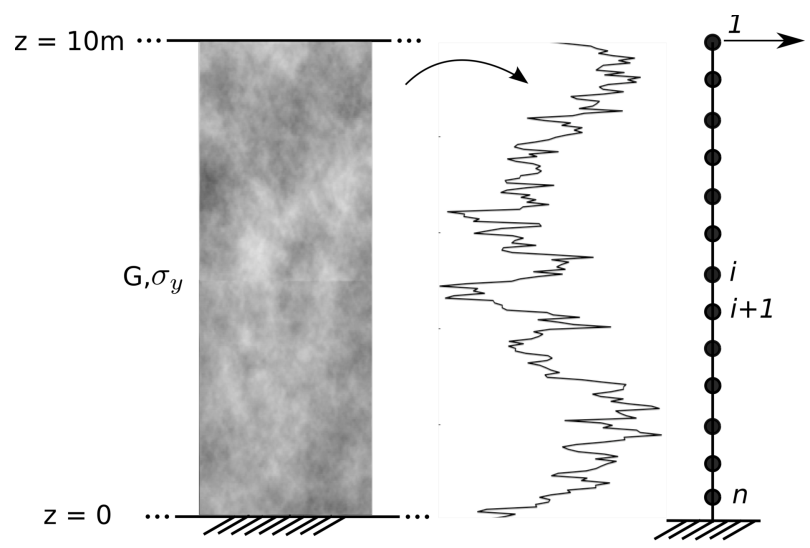

Figure 3: A realization of the stiffness random field and the idealized numerical model.

Fig. 4 shows the evolution of the PDF of stress at the first 4 orthogonal PC spaces at the top of the shear beam for case 1. Due to the small correlation length and large coefficient of variation of the shear modulus, all stress spaces are active. The evolution of the PDF in the mean shear stress space is initially diffusive and then sharpens in a quick transition to the elastoplastic regime. On the other hand, the remaining stress spaces exhibit mostly diffusion. The total reconstructed mean and standard deviation of stress vs the mean strain, 


\begin{tabular}{|c|c|c|c|c|c|}
\hline Case & $l_{c}^{G, \sigma_{y}}(\mathrm{~m})$ & $\langle G\rangle(\mathrm{MPa})$ & $C O V_{G}$ & $\left\langle\sigma_{y}\right\rangle(\mathrm{MPa})$ & $C O V_{\sigma_{y}}$ \\
\hline 1 & 0.2 & 50 & 0.4 & 0.8 & 0.1 \\
\hline 2 & 1 & 50 & 0.1 & 0.8 & 0.4 \\
\hline
\end{tabular}

\begin{tabular}{c|crrr} 
Case & $K_{P}(\mathrm{MPa})$ & $n_{K L}$ & $m_{P C_{d}}$ & $s_{P C_{k}}$ \\
\hline 1 & 0 & 2 & 2 & 2 \\
2 & 20 & 2 & 2 & 2
\end{tabular}

Table 1: Parameters for the examples in this study
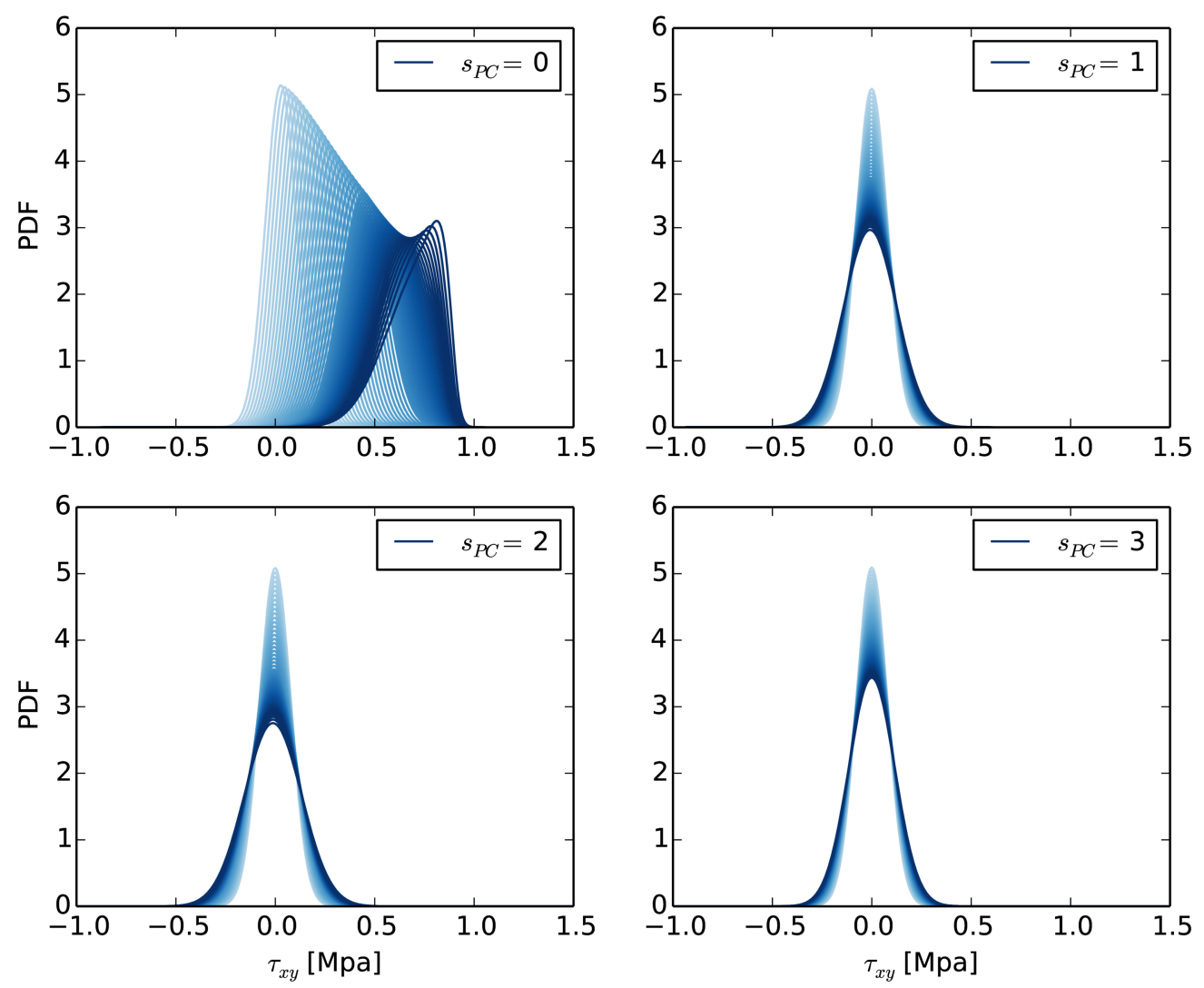

Figure 4: Evolution of the PDF of shear stress at the first 4 orthogonal PC spaces (Case $1)$. 


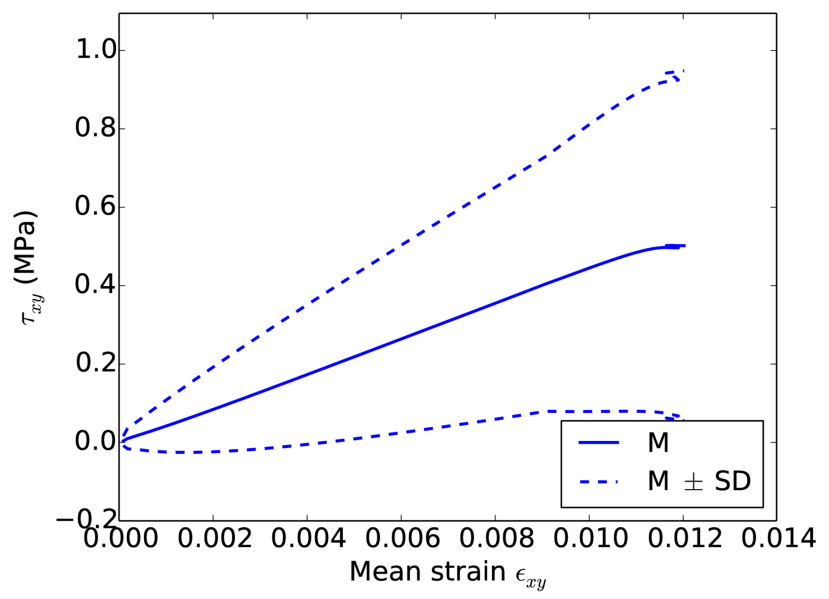

Figure 5: Mean $(\mathrm{M}) \pm$ standard deviation $(\mathrm{SD})$ of stress versus mean strain response at the top of the shear column (Case 1).

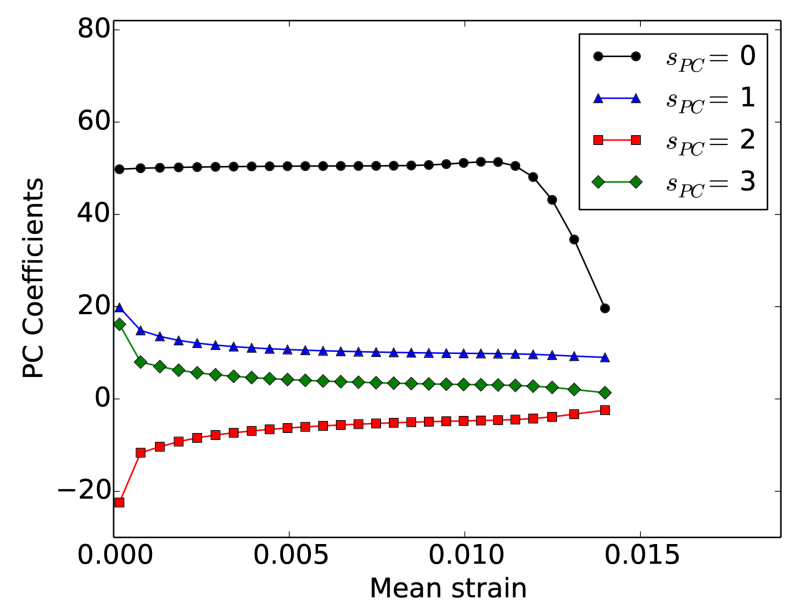

Figure 6: Evolution of PC coefficients of the linearized random shear stiffness (Case 1).

considering all PC terms, is plotted in Fig. 5, where the transition to the plastic regime is only evident towards the end of the simulation. At the same spatial point, Fig. 6 shows the evolution of PC coefficients derived by means 

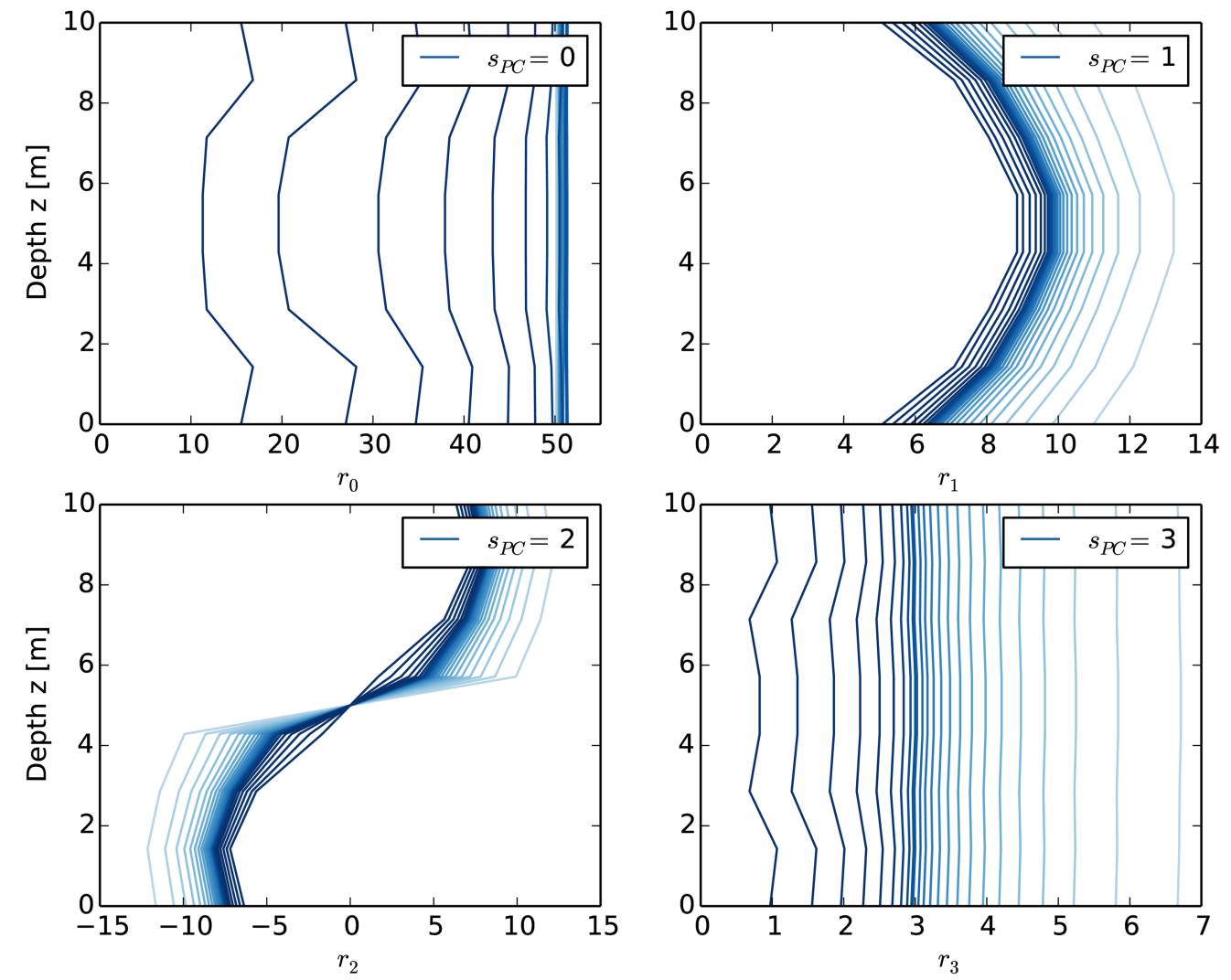

Figure 7: Evolution of the profile of PC coefficients of the linearized random shear stiffness along the depth of the shear beam (Case 1).

of the proposed linearization procedure. After a few steps, the optimization procedure has converged and the values of the coefficients remain almost constant for the elastic part of the response. Then, the $0^{\text {th }} \mathrm{PC}$ space exhibits a sharp decline towards zero, consistent with the mean stress response in an elastic-perfectly plastic body. The rest of the spaces decline at a smaller rate, in agreement with the evolution of second order stress statistics in those 


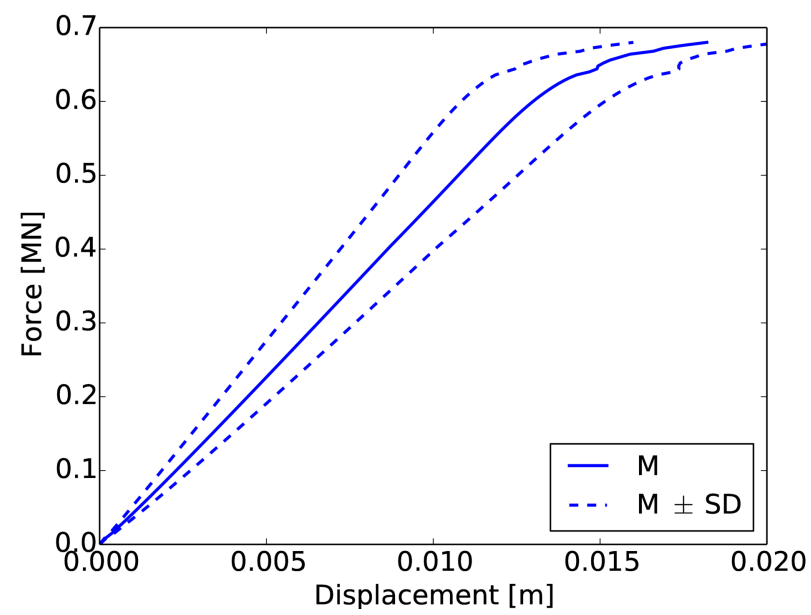

Figure 8: Force- mean $(\mathrm{M}) \pm$ standard deviation (SD) of displacement response at the top of the shear column (Case 1).

spaces towards the end of loading (see Fig. 4). The evolution of the profile of coefficients (along the depth of the shear beam) is given in Fig. 7, where the shape of the initial profile (light color) is determined by the underlying KL eigenvectors, while the accuracy is governed by the associated truncation error. It is evident that the aforementioned profile values ultimately tend to zero due to the elastic-perfectly plastic nature of the model. Finally, the global force versus (mean \pm standard deviation) displacement response at the top is shown in Fig. 8, where we can identify a sharp transition to a perfectly plastic response.

Case 2 involves a more uncertain initial yield strength along with a deterministic hardening modulus, which results in the characteristic evolution of the PDF of shear strength at the top as shown in Fig. 9. Due to the large correlation length and small coefficient of variation of the shear modulus, the 

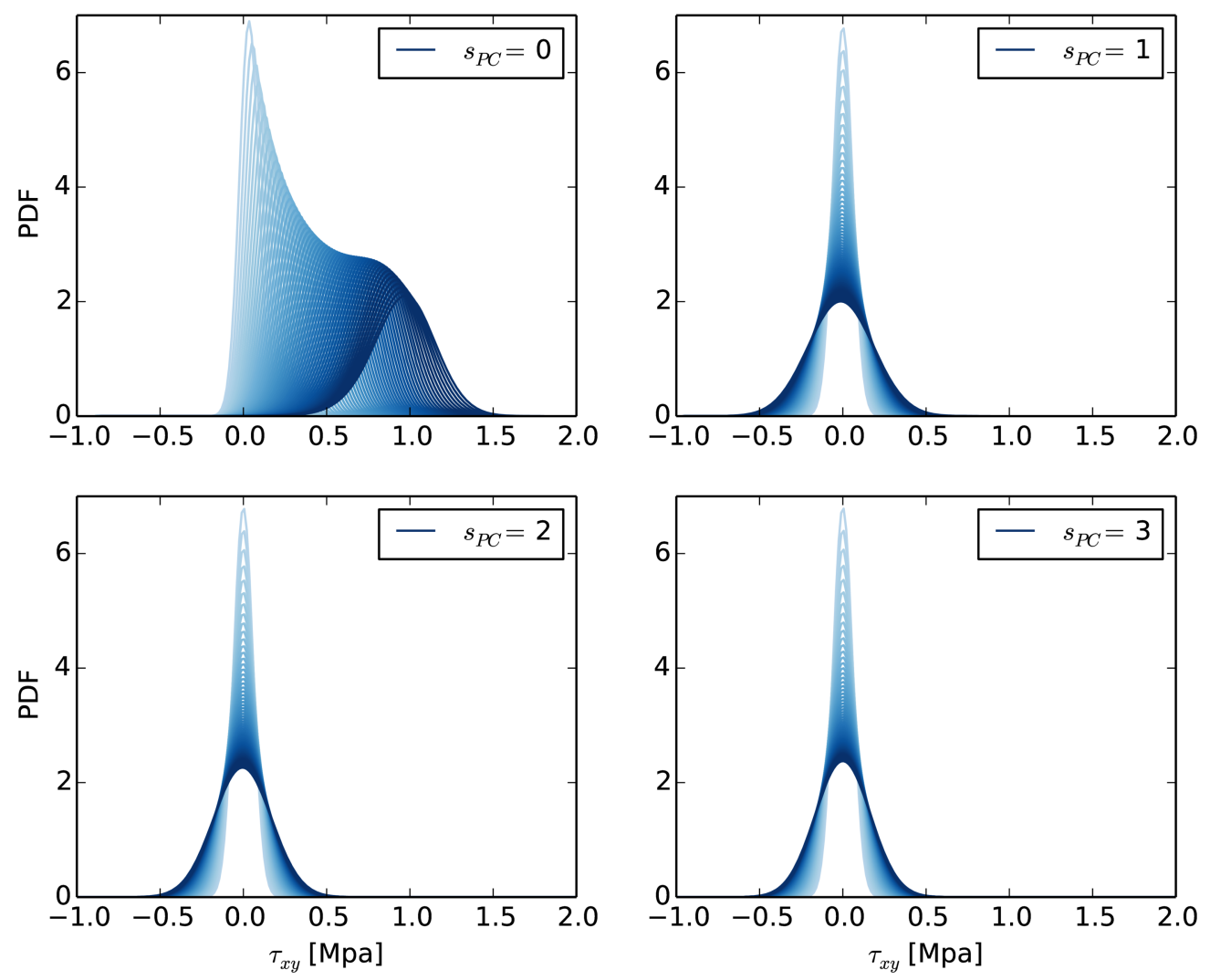

Figure 9: Evolution of the PDF of shear stress at the first 4 orthogonal KL/PC spaces (Case 2).

mean stress space is mostly active as well as the first stress space, which again is mostly diffusive. Fig. 10 shows the mean and standard deviation of the total stress versus the mean strain, at the same point, where a smooth transition to elastoplasticity is evident. The associated values of the PC coefficients are shown in Fig. 11, which again show a smooth decline of the governing coefficient due to the wide range of the elastoplastic transition. Fig. 12 shows the evolution of the profile of the PC coefficients of the linearized random 


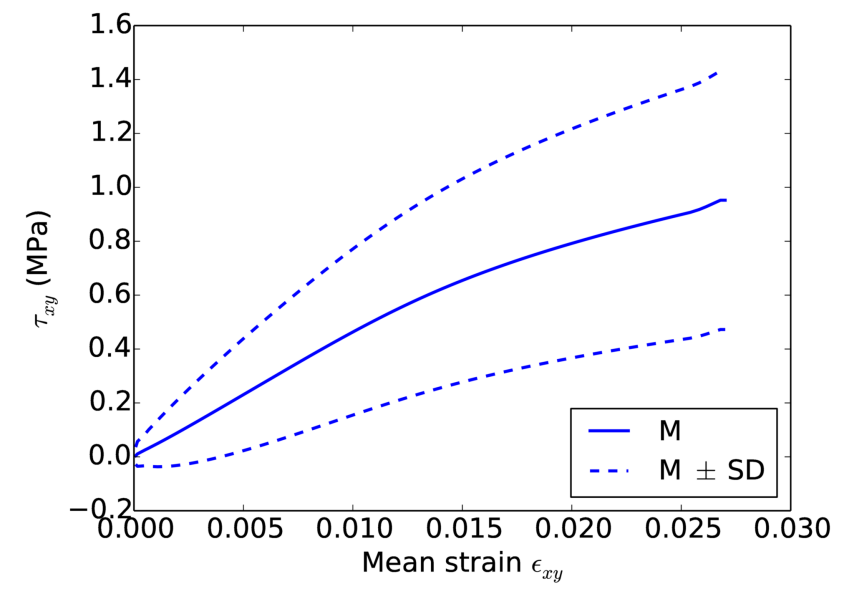

Figure 10: Mean $(\mathrm{M}) \pm$ standard deviation $(\mathrm{SD})$ of stress versus mean strain response at the top of the shear column (Case 2).

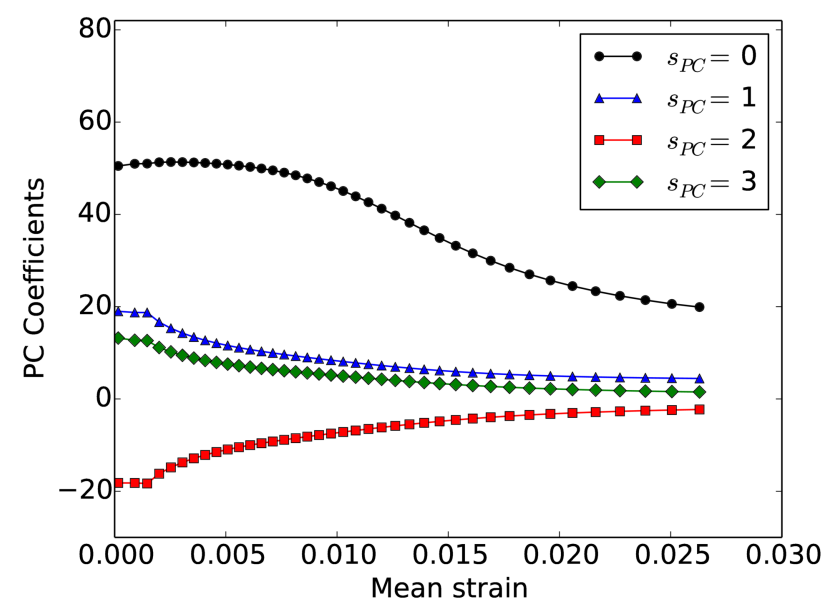

Figure 11: Evolution of PC coefficients of the linearized random shear stiffness (Case 2).

stiffness similar to Case 1. Finally the global force-displacement response at the top of the shear beam is shown in Fig. 13, where we can identify a smooth transition to a linear hardening response. 

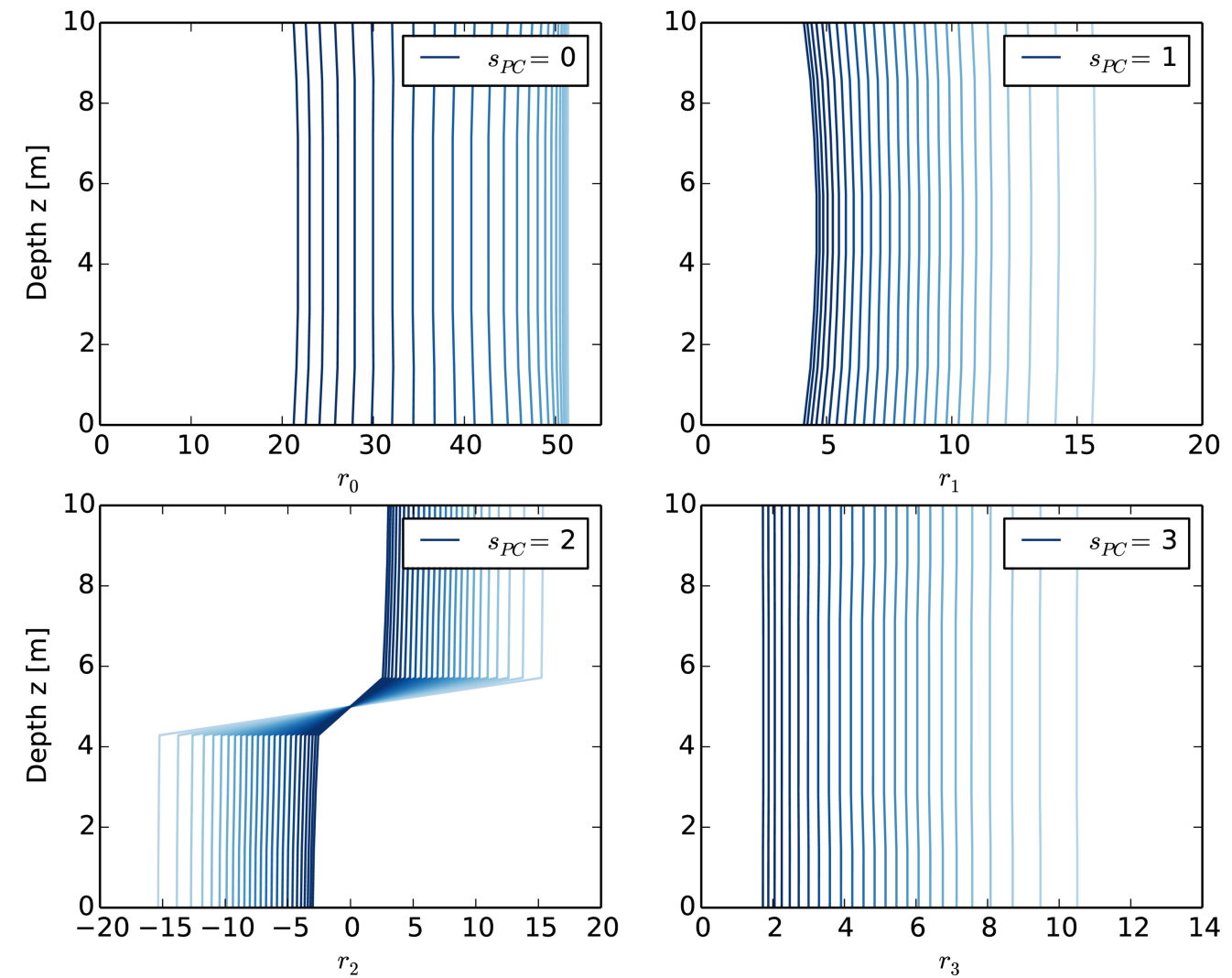

Figure 12: Evolution of the profile of PC coefficients of the linearized random shear stiffness along the depth of the shear beam (Case 2). 


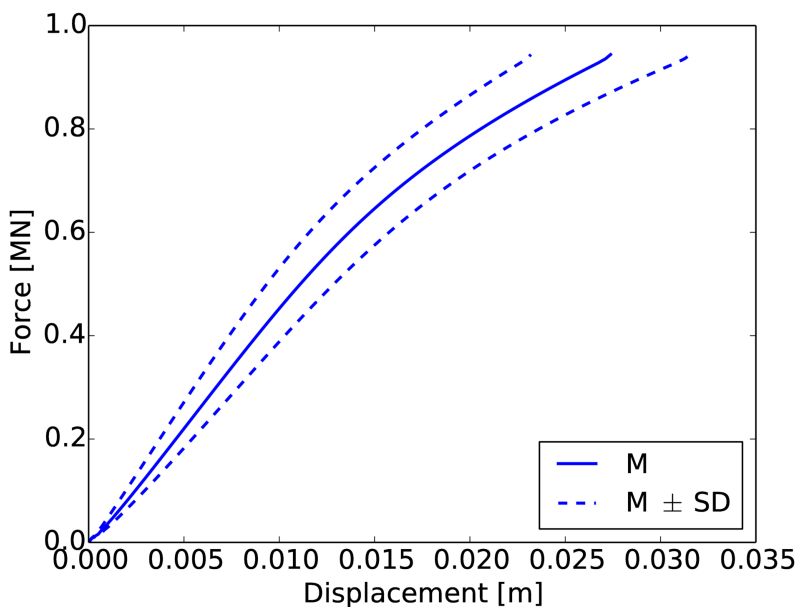

Figure 13: Force versus mean $(\mathrm{M}) \pm$ standard deviation $(\mathrm{SD})$ of displacement response at the top of the shear column (Case 2). 


\section{Conclusions}

We have proposed a numerical technique to solve inelastic random boundary value problems based on stochastic Galerkin techniques and a nonlocal Fokker-Planck-Kolmogorov plasticity framework. It relies on a general lin-

\section{Acknowledgements}

This work has been partly supported by the National Science Foundation under Award No. 1200702 as well as the Department of Civil and Environmental Engineering of the University of California, Davis. We are grateful 
425 to the anonymous reviewers for their constructive comments. 


\section{References}

[1] G. A. Fenton, Random field modeling of CPT data, ASCE Journal of Geotechnical and Geoenvironmental Engineering 125 (1999) 486-498.

[2] K.-K. Phoon, F. H. Kulhawy, Characterization of geotechnical variability, Canadian Geotechnical Journal 36 (1999) 612-624.

[3] A. L. Jones, S. L. Kramer, P. Arduino, Estimation of Uncertainty in Geotechnical Properties for Performance-Based Earthquake Engineering, Technical Report PEER 2002/16, Pacific Earthquake Engineering Research Center, 2002.

[4] G. I. Schüeller, A state-of-the-art report on computational stochastic mechanics, Probabilistic Engineering Mechanics 12 (1997) 197-321.

[5] H. G. Matthies, C. E. Brenner, C. G. Bucher, C. Guedes Soares, Uncertainties in probabilistic numerical analysis of structures and soilds stochastic finite elements, Structural Safety 19 (1997) 283-336.

[6] I. Babuska, N. F, R. Tempone, A stochastic collocation method for elliptic partial differential equations with random input data, SIAM J. Numer. Anal. 45 (2007) 1005-1034.

445

[7] D. Xiu, Fast numerical methods for stochastic computations, Commun. Comput. Physics 5 (2008) 242-272.

[8] S. Acharjee, N. Zabaras, A non-intrusive stochastic Galerkin approach for modeling uncertainty propagation in deformation processes, Computers \& Structures 85 (2007) 244-254. 
450

460

[12] R. G. Ghanem, P. D. Spanos, Stochastic Finite Elements: A Spectral Approach, Springer-Verlag, 1991. (Reissued by Dover Publications, 2003).

[13] N. Wiener, The homogeneous chaos, American Journal of Mathematics 465

[14] M. K. Deb, I. M. Babuska, J. T. Oden, Solution of stochastic partial differential equations using Galerkin finite element techniques, Computer Methods in Applied Mechanics and Engineering 190 (2001) 6359-6372.

[15] H. G. Matthies, A. Keese, Galerkin methods for linear and nonlinear 470 be intrusive? The solution of parametric and stochastic equations - the "plain vanilla" Galerkin case, SIAM J. Sci. Comput. 36 (2014) A2720A2744.

[10] A. Keese, A Review of Recent Developments in the Numerical Solution of Stochastic Partial Differential Equations (Stochastic Finite Elements), Scientific Computing 2003-06, Deapartment of Mathematics and Computer Science, Technical University of Braunschweig, Brunswick, Germany, 2003.

[11] H. Matthies, Uncertainty Quantification with Stochastic Finite Elements, Wiley, 2004. 60 (1938) 897-936.

elliptic stochastic partial differential equations, Computer Methods in

[9] L. Giraldi, A. Litvinenko, D. Liu, H. Matthies, A. Nouy, To be or not to Applied Mechanics and Engineering 194 (2005) 1295-1331. 
[16] D. Xiu, G. E. Karniadakis, The Wiener-Askey polynomial chaos for stochastic differential equations, SIAM Journal on Scientific Computing 24 (2002) 619-644.

[17] H. G. Matthies, A. Litvinenko, O. Pajonk, B. V. Rosić, E. Zander, Parametric and uncertainty computations with tensor product representations, in uncertainty quantification in scientific computing, IFIP Advances in Information and Communication Technology 377 (2012) $139-150$.

[18] A. Nouy, A. Cléent, eXtended stochastic finite element method for the numerical simulation of heterogeneous materials with random material interfaces, International Journal for Numerical Methods in Engineering 83 (2010) 1312-1344.

[19] M. Anders, M. Hori, Three-dimensional stochastic finite element method 485 for elasto-plastic bodies, International Journal for Numerical Methods in Engineering 51 (2001) 449-478.

[20] B. Jeremić, K. Sett, M. L. Kavvas, Probabilistic elasto-plasticity: Formulation in 1-D, Acta Geotechnica 2 (2007) 197-210.

[21] M. L. Kavvas, Nonlinear hydrologic processes: Conservation equations for determining their means and probability distributions, Journal of Hydrologic Engineering 8 (2003) 44-53.

[22] B. Jeremić, K. Sett, On probabilistic yielding of materials, Communications in Numerical Methods in Engineering 25 (2009) 291-300. 
[23] K. Sett, B. Jeremić, M. L. Kavvas, Stochastic elastic-plastic finite elements, Computer Methods in Applied Mechanics and Engineering 200 (2011) 997-1007.

[24] B. Rosić, A Review of Computational Stochastic Elastoplasticity, Technical Report Informatikbericht Nr. 2008-08, Technische Universität Braunschweig, 2009.

[25] M. Arnst, R. Ghanem, A variational-inequality approach to stochastic boundary value problems with inequality constraints and its application to contact and elastoplasticity, International Journal for Numerical Methods in Engineering 89 (2012) 1665-1690.

[26] S. Sakamoto, R. Ghanem, Polynomial chaos decomposition for the simulation of non-Gaussian nonstationary stochastic processes, Journal of Engineering Mechanics 128 (2002) 190-201.

[27] F. Benth, J. Gjerde, Convergence rates for finite element approximations for stochastic partial differential equations, Stochastics: An International Journal of Probability and Stochastic Reports 63 (1998) 313-326.

[28] C. Soize, R. Ghanem, Physical systems with random uncertainties: Chaos representations with arbitrary probability measure, SIAM Journal on Scientific Computing 26 (2004) 395-410.

[29] M. Rosenblatt, Remarks on a multivariate transformation, The Annals of Mathematical Statistics 23 (1952) 470-472.

[30] A. Nataf, Dètermination des distributions de probabilitès dont les 
marges sont donnèes, Comptes Rendus de l'Acadèmie des Sciences 225 (1962) 42-43.

[31] R. Ghanem, The nonlinear Gaussian spectrum of log-normal stochastic processes and variables, J. Appl. Mech 66 (1966) 964-973.

[32] I. Babuska, P. Chatzipantelidis, On solving elliptic stochastic partial differential equations, Computer Methods in Applied Mechanics and Engineering 191 (2002) 4093 - 4122.

[33] R. Courant, D. Hilbert, Methods of Mathematical Physics, Wiley, 1989.

[34] K. Phoon, S. Huang, S. Quek, Implementation of Karhunen-Loeve expansion for simulation using a wavelet-Galerkin scheme, Probabilistic Engineering Mechanics 17 (2002) 293-303.

[35] B. Khoromskij, A. Litvinenko, H. Matthies, Application of hierarchical matrices for computing the Karhunen-Love expansion, Computing 84 (2009) 49-67.

[36] S. Rahman, H. Xu, A meshless method for computational stochastic mechanics, Int. J. Comput. Meth. Eng. Sc. Mech. 6 (2005) 41-58.

[37] A. Der Kiureghian, B. J. Ke, The stochastic finite element method in structural reliability, Journal of Probabilistic Engineering Mechanics 3 (1988) 83-91.

535

[38] C.-C. Li, A. Der Kiureghian, Optimal discretization of random fields, Journal of Engineering Mechanics, ASCE 119 (1993) 1136-1154. 
[39] T. Melink, J. Korelc, Stability of Karhunen-Loeve expansion for the simulation of gaussian stochastic fields using galerkin scheme, Probabilistic Engineering Mechanics 37 (2014) 7 - 15.

540

[40] T. Hughes, The Finite Element Method: Linear Static and Dynamic Finite Element Analysis, Prentice Hall Inc., 1987.

[41] Wolfram Research Inc., Mathematica Version 5.0, Wolfram Research Inc., Champaign, Illonois, 2003.

[42] R. Ghanem, R. M. Kruger, Numerical solution of spectral stochastic finite element systems, Computer Methods in Applied Mechanics and Engineering 129 (1996) 289 - 303.

[43] E. Ullmann, Solution strategies for stochastic finite element discretizations, Doctoral dissertation, TU Bergakademie Freiberg, 2008.

[44] O. L. Maitre, O. M. Knio, Spectral methods for uncertainty quantification with applications to computational fluid dynamics, Springer, Dordrecht, 2010.

[45] D. Ghosh, P. Avery, C. Farhat, A FETI-preconditioned conjugate gradient method for large-scale stochastic finite element problems, International Journal for Numerical Methods in Engineering 80 (2009) 914-931.

[46] K. Levenberg, A method for the solution of certain non-linear problems in least squares, Quart. Appl. Math. 2 (1944) 164-168. 
*Biographies of authors

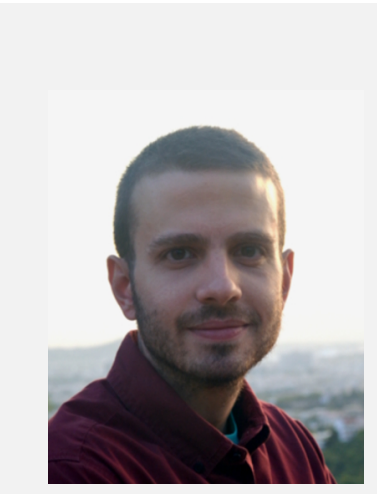

\section{Konstantinos Karapiperis}

Konstantinos Karapiperis earned his Diploma from the National Technical University of Athens, Greece and his M.S. from the University of California, Davis, both in civil and environmental engineering. He has been part of the Computational Geomechanics Group at UC Davis and the Soil Mechanics Laboratory at NTUA. His research interests include the inelastic modeling and uncertainty quantification of the behavior of heterogeneous materials and civil engineering systems. 


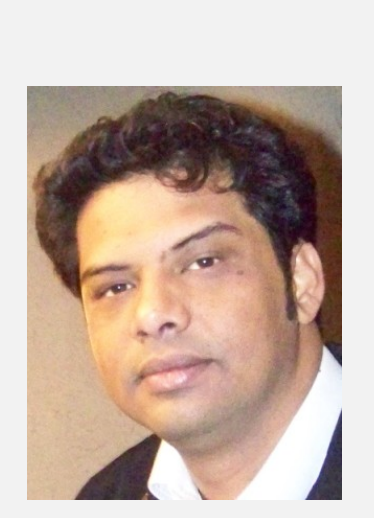

\section{Kallol Sett}

Kallol Sett, Assistant Professor of Civil, Structural and Environmental Engineering at the University at Buffalo, The State University of New York, earned his bachelor's degree with honors from Jadavpur University, Calcutta, India in 1997, his master's degree from the University of Houston in 2003, and his doctorate from the University of California, Davis, all in civil engineering. He has previously taught at the University of Akron, Ohio and has worked for the California Department of Transportation as well as Afcons Infrastructure Limited in Bombay, India. His research interests are in assessment and mitigation of risks in civil engineering constructed facilities. 


\section{Boris Jeremić}

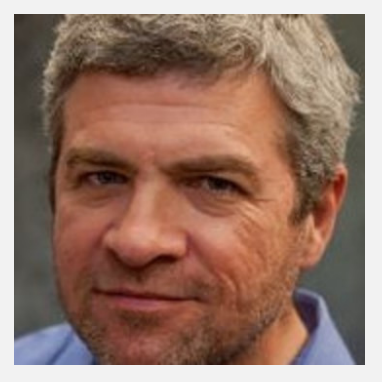

Boris Jeremic is a Professor of Civil and Environmental Engineering at the University of California at Davis, a Faculty Scientist at the Earth Science Division of the Lawrence Berkeley National Laboratory as well as a visiting Professor at the University of Kragujevac and the University of Belgrade. He earned his Diploma from Belgrade University and his M.S and PhD from the University of Colorado at Boulder, all in Civil Engineering. His research interests are related to the computational modeling and simulation of static and dynamic inelastic behavior of engineering solids and structures with emphasis on geomechanics. 


\section{Levent Kavvas}

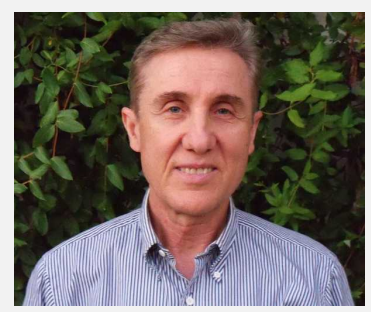

M. Levent Kavvas is the Gerald and Lilian Orlob Endowed Chair Professor of Water Resources Engineering in the Department of Civil and Environmental Engineering at the University of California at Davis. He earned his M.S. degree from Colorado State University at Ft. Collins and his Ph.D. from Purdue University, both in civil engineering. His areas of specialization include the theory of stochastic processes and the mathematical modeling of the integrated hydrologic-atmospheric processes at multiple scales for the simulation and prediction of hydrologic water balances toward quantifying phenomena such as floods and droughts. He is the author of more than 220 papers in stochastic processes, hydrology and hydrometeorology. 